Reprod. Nutr. Dévelop., 1986, 26 (1 B), 349-350.

\title{
Influence de la nature de l'aliment concentré sur le métabolisme des chèvres en fin de gestation et en début de lactation
}

\author{
P. MORAND-FEHR, P, BAS, D. SAUVANT, J. HERVIEU, Y. CHILLIARD
}

Station de Nutrition et Alimentation, I.N.R.A. Institut National Agronomique Paris-Grignon 16 rue Claude Bernard 75231 Paris Cédex 05

Summary. From 8 weeks before to 8 weeks after parturition, 3 groups of 6 dairy goats were given lucerne hay, maize silage and concentrate rich in starch $(S)$ or fiber $(F)$ or protected lipids $(L)$. The values of blood parameters are the same with diets $A$ and $P$ but diet $L$ decreased the energy balance of goats.

Matériel et méthodes. L'effet de la nature de l'aliment concentré sur les paramètres zootechniques et métaboliques a été suivi sur 18 chèvres laitières réparties en 3 lots de la $8^{e}$ semaine avant $(S-8)$ à la $8^{e}$ semaine après la parturition $(S+8)$. Elles reçoivent ad lib. individuellement du foin de luzerne et de l'ensilage de maïs et de façon rationnée l'un des 3 concentrés $A, P$ et $L$, respectivement riches en amidon, en glucides pariétaux et en lipides (lipides protégés Alta lipids) (teneurs en sucres + amidon : $41,5,18,5$ et $26,6 \mathrm{~g} / 100 \mathrm{~g} \mathrm{M.S}$. ; en ADF : 6,7 , 16,8 et 13,4 ; en extrait éthéré : $2,5,2,7$ et 7,4 respectivement).

Résultats. Le régime $L$ réduit légèrement les consommations de concentré et de fourrages, significativement de $S+2$ à $S+8$ (tabl. 1). La production de lait n'est pas significativement différente mais la teneur du lait en matières grasses est plus élevée avec le régime $L$. Les paramètres de consommation, de production et de composition du lait des lots $A$ et $P$ sont très proches. Les teneurs plasmatiques en $A G N E$ et $\beta$-hydroxybutyrate $(B H B)$ des lots $A$ et $P$ sont très voisines alors que

TABL. 1. - Influence de la nature du concentré sur la consommation et la production et la composition du lait de chèvre.

\begin{tabular}{|c|c|c|c|c|}
\hline \multirow[b]{2}{*}{ Type de concentré } & \multicolumn{4}{|c|}{ Lactation } \\
\hline & $\mathrm{A}$ & $\mathrm{P}$ & $\mathrm{L}$ & Sign. (1) \\
\hline M.S.T. ingérée $\mathrm{Kg} / \mathrm{j}$ & 2,19 & 2,19 & 1,82 & * \\
\hline M.S. Fourrages $\mathrm{Kg} / \mathrm{j}$ & 1,34 & 1,36 & 1,13 & * \\
\hline M.S. Concentré Kg/j & 0,75 & 0,83 & 0,68 & N.S. \\
\hline Bilan énergétique ${ }^{(2)} \mathrm{Kcal} / \mathrm{j}$ & -496 & -642 & -980 & * \\
\hline Production lait $\mathrm{Kg} / \mathrm{j}$ & 2,95 & 3,22 & 3,25 & N.S. \\
\hline Teneur du lait en M.G. \% & 5,10 & 4,81 & 5,70 & $* *$ \\
\hline Teneur du lait en M.A. \% & 3,98 & 3,71 & 3,65 & * \\
\hline$C_{18}: 0+C_{18}: 1 \%$ & 31,3 & 30,1 & 41,3 & $* * *$ \\
\hline
\end{tabular}

(1) N.S. non significatif; * significatif à $\mathrm{P} \leq 0,05$; ** significatif à $\mathrm{P} \leq 0,01$; ** significatif à $P \leq 0,001$.

(2) Bilan énergétique en énergie nette $=$ Energie nette des aliments ingérés - Dépenses d'énergie nette d'entretien et de lactation. 
celles du lot $L$ sont le plus souvent significativement supérieures (fig. 1) ce qui indiquerait que la mobilisation des lipides des tissus adipeux est plus importante dans le lot $L$. Le bilan énergétique plus faible et le pourcentage plus élevé d'acides en $\mathrm{C}_{18}$ dans la matière grasse du lait le confirment (tabl. 1). Cette mobilisation plus intense est due très probablement au moindre niveau de consommation qui est très propablement liée à une protection des lipides insuffisante (COOP communication personnelle).

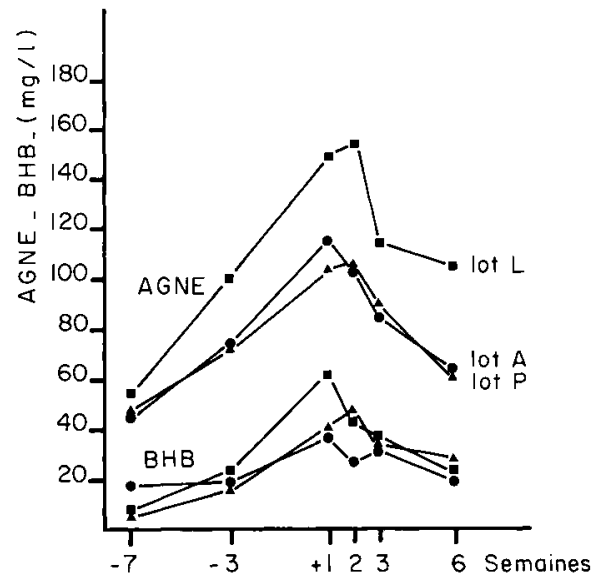

FIG. 1. - Paramètres sanguins

Mais un apport supplémentaire de lipides en pleine lactation tend à augmenter le prélèvement des triglycérides de lipoprotéines, la teneur plasmatique en AGNE et le pourcentage des acides en $\mathrm{C}_{18}$ de la matière grasse du lait (Bines et al., 1978). L'apport de lipides dans la présente expérience semble avoir aussi modifié ces paramètres, d'autant plus que la lactation progresse. Les corrélations entre la quantité de lipides ingérés et la teneur plasmatique en AGNE (significatives à partir de $S+6$ ) ou le pourcentage en $C_{18}$ dans la matière grasse du lait (significatives à partir de $S+2$ ) deviennent de plus en plus étroites.

La teneur plasmatique en $\mathrm{BHB}$ du lot $L$ est très proche de celle des lots $P$ et $A$ en $S+6$, alors que la teneur en $A G N E$ et le pourcentage en acides $C_{18}$ dans le lait sont significativement plus élevés. Cela tendrait à indiquer que tous les bilans énergétiques étant encore négatifs, la teneur en AGNE n'est plus seulement le reflet de la mobilisation des tissus adipeux mais aussi celui de l'apport de lipides alimentaires.

Ainsi les régimes en glucides pariétaux et en amidon présentent très peu de différences entre eux (toujours non significatives) sur les paramètres métaboliques la production et la composition du lait. En revanche, l'addition de matière grasse sous forme de lipides protégés au régime favorise la sécrétion de matière grasse de la mamelle en début de lactation et modifie les paramètres qui sont considérés comme le reflet de la mobilisation des réserves adipeuses en début de lactation et qui de ce fait acquièrent une signification métabolique différente.

Bines J. A., Brumby P. E., Storry J. E., Fulford R. J., Braithwaite G. D., 1978. The effect of protected lipids on nutrient intake blood and rumen metabolites and milk secretion in dairy cows during early lactation. J. agric. Sci. (Camb.), 91, 135-150. 